\title{
mHealth Interventions for Self-Harm: Scoping Review
}

Bethany Cliffe ${ }^{1}, \mathrm{BSc}, \mathrm{MSc}$; Jessica Tingley ${ }^{2 *}, \mathrm{BSc}, \mathrm{MSc}$; Isobel Greenhalgh ${ }^{2 *}, \mathrm{BSc}$, MRes; Paul Stallard ${ }^{1,2}, \mathrm{PhD}$

${ }^{1}$ Department for Health, University of Bath, Bath, United Kingdom

${ }^{2}$ Child and Adolescent Mental Health Services, Oxford Health NHS Foundation Trust, Bristol, United Kingdom

* these authors contributed equally

Corresponding Author:

Bethany Cliffe, BSc, MSc

Department for Health

University of Bath

Claverton Down

Bath,

United Kingdom

Phone: 4401225388388

Email: bc731@bath.ac.uk

\section{Abstract}

Background: Self-harm is a growing issue with increasing prevalence rates; however, individuals who self-harm do not often receive treatment. Mobile health (mHealth) interventions are a possible solution to some of the barriers that individuals face when seeking support, and they have also been found to be effective in improving mental health. Thus far, reviews of mHealth interventions for self-harm have been limited by study type. Therefore, we determined that a broader scoping review will provide a more exhaustive understanding of mHealth interventions for self-harm.

Objective: This scoping review aims to identify mHealth interventions for self-harm within the literature, understand the types and features of interventions that have been developed and evaluated, highlight research findings around mHealth interventions for self-harm, and determine what outcomes are typically used to assess the efficacy of interventions.

Methods: A search was conducted using Embase, PubMed, PsycINFO, PsycEXTRA, Web of Science, and the Cochrane Library. Studies were included if they described an mHealth intervention designed to have a direct (ie, if the intervention was designed for self-harm or for people who self-harm) or indirect (ie, if self-harm was measured as an outcome) treatment effect and if the paper was available in English. There were no exclusion criteria based on the study design.

Results: A total of 36 papers were included in the review, and most of them were randomized controlled trials published within the last 4 years. The interventions were mostly smartphone apps and calling or texting services, with 62\% (21/34) having underlying therapeutic models to inform the intervention content. They were generally shown to be promising and appealing, but only 5 were widely available for use. Outcomes focused on a reduction of self-harm and suicidality, mood, and the users' experiences of the intervention. Samples were typically nondiverse, and there was limited variety in the study designs and in the measurements of self-harm recovery.

Conclusions: Promising and appealing mHealth interventions have been developed but are not widely available. Research could benefit from greater diversity as well as a broader and more nuanced understanding of recovery from self-harm.

(J Med Internet Res 2021;23(4):e25140) doi: 10.2196/25140

\section{KEYWORDS}

mHealth; self-harm; digital interventions; self-injury; NSSI; mobile phone

\section{Introduction}

\section{Self-harm}

The National Institute for Health and Care Excellence defines self-harm as any act of self-injury or poisoning, irrespective of the motivation behind the act [1]. It is a growing concern that can have great physical, psychological [2], and societal [3] costs. Notably, self-harm has been identified as a significant and persistent predictor of suicide [4]. In recent years, the lifetime prevalence of self-harm in the general English population has seen a sharp increase from $2.4 \%$ in 2000 to $6.4 \%$ in 2014; this was most common in young adult females who reported an increase in prevalence from $6.5 \%$ to $19.7 \%$ [5]. Furthermore, 
given that individuals are often reluctant to disclose their self-harm behaviors due to the shame and stigma associated with it [6], rates of self-harm may be even higher than what these figures suggest [7].

\section{Help for Self-harm}

Tørmoen et al [8] surveyed 11,440 young people aged 14-17 years in Norway and found that only $34 \%$ of those who had self-harmed had ever sought professional help, indicating that help-seeking is low among those who self-harm $[9,10]$. Concerns over being perceived as "attention-seeking" or "crazy" and difficulty talking about their self-harm behaviors have been identified as some of the barriers to seek support by adolescents [11]. Furthermore, a lack of effective interventions for self-harm creates barriers to receive support when an individual seeks professional help. The National Institute for Health and Care Excellence guidelines advise against the use of pharmacological treatments for self-harm, instead recommending psychological interventions tailored to self-harm that may involve problem solving, cognitive behavioral or psychodynamic elements [12]. Despite this, to date, there is limited high-quality evidence suggesting that psychological or pharmacological interventions for self-harm are effective $[13,14]$. Moreover, increased pressure on services and the resulting difficulties with the availability and accessibility of these interventions can further prevent individuals from receiving support [15].

\section{Use of Mobile Health}

The use of mobile health (mHealth) may help overcome the barriers to treatment accessibility and availability. mHealth is a branch of eHealth, defined by the Global Observatory for eHealth as "medical and public health practice supported by mobile devices, such as mobile phones, patient monitoring devices, personal digital assistants (PDAs), and other wireless devices" [16]. Given the ubiquity of mobile phone ownership $[17,18]$, providing mental health support in this way has the potential to reach many individuals who may not be receiving help for self-harm. mHealth offers multiple possibilities, including self-help smartphone apps, SMS text messaging with a support service, physical symptom tracking through wearable technologies, and receiving virtual therapy [19]. Clough and Casey [20] found that mHealth users felt that receiving virtual mHealth therapy was more beneficial compared with face-to-face therapy, particularly highlighting the freedom they felt to be completely open and honest with their therapist. mHealth tools also have merit as standalone interventions, with some studies reporting reductions in symptoms of mental health difficulties, including anxiety [21], schizophrenia [22], depression [23], and borderline personality disorder [24].

Studies investigating the efficacy of mHealth interventions for managing self-harm have also been reviewed but they are limited. Witt et al [25] identified only one study that included outcome measures of an mHealth intervention for self-harm, whereas Melia et al [26] identified 2. These reviews focused on randomized controlled trials (RCTs) and pre- and poststudies. Arshad et al [27] focused more closely on self-harm and identified 22 studies; however, this was still limited by their decision to exclude qualitative studies and those where self-harm was not the primary outcome. A broader scoping review will help to identify more mHealth tools available for managing self-harm and broaden our knowledge of them.

\section{Aims}

This scoping review aims to (1) identify mHealth interventions for self-harm within the literature, (2) understand the types and features of interventions that have been developed and evaluated, (3) highlight research findings around mHealth interventions for self-harm, and (4) determine what outcomes are typically used to assess the efficacy of interventions.

\section{Methods}

\section{Overview}

A detailed methodology can be found in the review protocol [28]. The following databases were searched in April 2020: Embase, PubMed, PsycINFO, PsycEXTRA, Web of Science, and the Cochrane Library. The reference lists of all papers identified in the searches were also screened. Multimedia Appendix 1 describes the full and detailed search strategy.

After duplicates were removed, the titles and abstracts were initially screened according to the aims of this review and were progressed for a full screening if they met the following inclusion criteria: (1) the study described an mHealth intervention (eg, SMS text messaging, phone calls, or websites accessible through a mobile device) designed to have a direct (ie, if the intervention was designed for self-harm or for people who self-harm) or indirect (if self-harm was measured as an outcome) treatment effect and (2) the paper was in English.

The full texts of the papers that met these criteria were then screened. Before screening the title and abstract, a pilot screening was performed by all 3 reviewers (BC, JT, and IG) on 20 papers selected at random. An interrated reliability check of at least $75 \%$ agreement was required to progress the papers to full screening. Initially, $80 \%$ agreement was achieved, and the remaining papers were briefly discussed until $100 \%$ agreement was achieved. Each paper was screened by at least two reviewers, with a third reviewer resolving any inconsistencies. During the title and abstract screening, there was $98 \%$ agreement between the 2 reviewers on each paper, with the others being discussed and resolved again. The full texts of the progressed papers were screened for eligibility.

\section{Data Charting Process}

The reviewers extracted predefined data regarding the study details (eg, year and country), participants (eg, number, age, and ethnicity), type of mHealth intervention, study design, measures, and outcomes. Both before and after data extraction, consistency was checked between reviewers using a random sample of papers. Data extraction was an iterative process in which categories were added or amended in accordance with the aims of the review. 


\section{Results}

\section{Identify mHealth Interventions for Self-Harm Within the Literature}

The search results are summarized in Figure 1. A total of 295 papers were identified. After duplicates were removed, 78\% $(229 / 295)$ of titles and abstracts were left to be screened. About $35 \%(79 / 229)$ of titles and abstracts progressed to a full-text screening, resulting in 54\% (43/79) of papers being excluded.
Of these, 51\% (22/43) did not present an mHealth intervention for self-harm, $21 \%$ (9/43) were systematic reviews that did not identify any papers not already identified by our search, and $19 \%(8 / 43)$ were protocols for studies for which a full text had since become available. Of the remaining 4 papers, $2(5 \%)$ were replies or comments on other studies, 1 (2\%) was a content analysis of apps that were commercially available with no references to an evidence base, and 1 (2\%) was a description of an app that had already been identified. A total of 36 papers met the inclusion criteria and are summarized in Table 1.

Figure 1. Flowchart of search results. mHealth: mobile health.

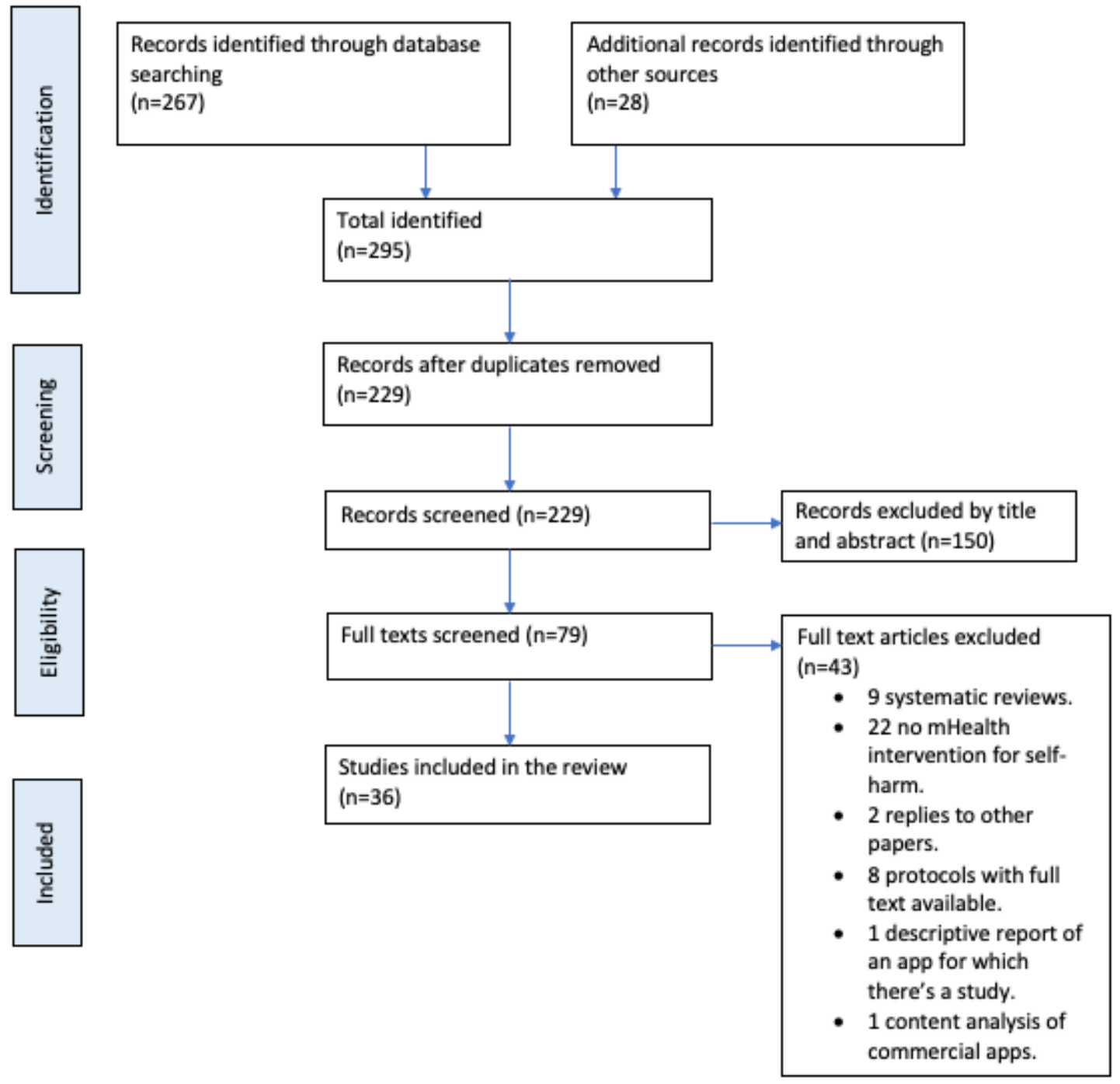


Table 1. Summary of papers $(\mathrm{N}=36)$.

\begin{tabular}{|c|c|c|c|c|c|c|c|c|}
\hline $\begin{array}{l}\text { Intervention de- } \\
\text { tails }\end{array}$ & $\begin{array}{l}\text { Type of inter- } \\
\text { vention }\end{array}$ & Design & Sample & $\begin{array}{l}\text { Type of self- } \\
\text { harm }\end{array}$ & $\begin{array}{l}\text { Self-harm measure } \\
\text { used }\end{array}$ & Development & Availability & $\begin{array}{l}\text { Improve- } \\
\text { ment in } \\
\text { self-harm }\end{array}$ \\
\hline $\begin{array}{l}\text { SIAM (2014) } \\
{[30]: 9 \text { texts sent, }} \\
\text { staggered for } 6 \\
\text { months following } \\
\text { A\& } E^{c} \text { discharge }\end{array}$ & $\begin{array}{l}\text { Supportive and } \\
\text { monitoring } \\
\text { texts }\end{array}$ & $\begin{array}{l}\mathrm{RCT} \text { (pro- } \\
\text { tocol) }\end{array}$ & $\begin{array}{l}\text { Adults dis- } \\
\text { charged after } \\
\text { suicide at- } \\
\text { tempt-France }\end{array}$ & $\begin{array}{l}\text { Suicidal self- } \\
\text { harm }\end{array}$ & $\begin{array}{l}\text { Columbia-Suicide } \\
\text { Severity Rating } \\
\text { Scale }\end{array}$ & $\begin{array}{l}\text { Developed by the } \\
\text { authors }\end{array}$ & $\begin{array}{l}\text { Not widely } \\
\text { available }\end{array}$ & No data \\
\hline $\begin{array}{l}\text { EpxDepression } \\
(2017) \text { [33]: Dai- } \\
\text { ly EMA }{ }^{d} \text { for } 2-4 \\
\text { months }\end{array}$ & $\begin{array}{l}\text { Text-based } \\
\text { EMA that noti- } \\
\text { fies care team } \\
\text { of risk }\end{array}$ & $\begin{array}{l}\text { Proof of } \\
\text { concept }\end{array}$ & $\begin{array}{l}\text { Adoles- } \\
\text { cents-United } \\
\text { States }\end{array}$ & $\begin{array}{l}\text { Suicidal self- } \\
\text { harm }\end{array}$ & $\begin{array}{l}\text { Last question of } \\
\mathrm{PHQ}^{\mathrm{e}-9}\end{array}$ & $\begin{array}{l}\text { Clinicians, re- } \\
\text { searchers, and } \\
\text { biostatisticians } \\
\text { with feedback } \\
\text { from target end } \\
\text { users }\end{array}$ & $\begin{array}{l}\text { Available for } \\
\text { health care } \\
\text { providers on } \\
\text { subscription }\end{array}$ & Yes \\
\hline $\begin{array}{l}\text { Imaginator } \\
(2020)[34]: \text { Ac- } \\
\text { cessible anytime }\end{array}$ & $\begin{array}{l}\text { Smartphone app } \\
\text { to promote self- } \\
\text { management }\end{array}$ & $\mathrm{RCT}$ & $\begin{array}{l}\text { Youth (aged 16- } \\
25 \text { years) cur- } \\
\text { rently self- } \\
\text { harming-United } \\
\text { Kingdom }\end{array}$ & $\begin{array}{l}\text { Suicidal and } \\
\text { nonsuicidal } \\
\text { self-harm }\end{array}$ & $\begin{array}{l}\text { Strength of motiva- } \\
\text { tion for reducing } \\
\text { self-harm scale, } \\
\text { craving experience } \\
\text { questionnaire for } \\
\text { self-harm, self- } \\
\text { harm imagery inter- } \\
\text { view. Self-harm, } \\
\text { frequency, severi- } \\
\text { ty, and self-effica- } \\
\text { cy for control were } \\
\text { measured with } \\
\text { items developed by } \\
\text { the enhancer }\end{array}$ & $\begin{array}{l}\text { Codeveloped } \\
\text { with youth with } \\
\text { lived experience } \\
\text { of self-harm }\end{array}$ & $\begin{array}{l}\text { Not widely } \\
\text { available }\end{array}$ & Yes \\
\hline $\begin{array}{l}\text { Therapeutic Eval- } \\
\text { uative Condition- } \\
\text { ing (2016) [35]: } \\
\text { Accessible any- } \\
\text { time }\end{array}$ & $\begin{array}{l}\text { Smartphone app } \\
\text { to decondition } \\
\text { self-harm }\end{array}$ & RCT & $\begin{array}{l}\text { Adults with } \\
\text { self-harm-inter- } \\
\text { national }\end{array}$ & $\begin{array}{l}\text { Suicidal and } \\
\text { nonsuicidal } \\
\text { self-harm }\end{array}$ & $\begin{array}{l}\text { Self-injurious } \\
\text { thoughts and behav- } \\
\text { iors interview }\end{array}$ & $\begin{array}{l}\text { Developed by the } \\
\text { authors }\end{array}$ & $\begin{array}{l}\text { No longer } \\
\text { available }\end{array}$ & $\begin{array}{l}\text { Yes, but } \\
\text { did not } \\
\text { persist at } \\
\text { follow-up }\end{array}$ \\
\hline $\begin{array}{l}\text { BlueIce }(2018) \\
{[36,37]: \text { Accessi- }} \\
\text { ble anytime }\end{array}$ & $\begin{array}{l}\text { Smartphone app } \\
\text { to help manage } \\
\text { urges to self- } \\
\text { harm }\end{array}$ & $\begin{array}{l}\text { Pre-post } \\
\text { phase } 1 \\
\text { trial }\end{array}$ & $\begin{array}{l}\text { Adolescents } \\
\text { currently self- } \\
\text { harming or at } \\
\text { risk of self- } \\
\text { harming attend- } \\
\text { ing } \\
\text { CAMHS } \\
\text { ed Kingdom }\end{array}$ & $\begin{array}{l}\text { Nonsuicidal } \\
\text { self-harm }\end{array}$ & $\begin{array}{l}\text { Semistructured in- } \\
\text { terview; Self Harm } \\
\text { data from clinical } \\
\text { records }\end{array}$ & $\begin{array}{l}\text { Coproduced with } \\
\text { youth with lived } \\
\text { experience of } \\
\text { self-harm, with } \\
\text { input from clini- } \\
\text { cal staff, aca- } \\
\text { demics, and app } \\
\text { developers }\end{array}$ & $\begin{array}{l}\text { Available for } \\
\text { CAMHS on } \\
\text { subscription }\end{array}$ & Yes \\
\hline $\begin{array}{l}\text { Unnamed (2018) } \\
\text { [38]: Accessible } \\
\text { anytime }\end{array}$ & $\begin{array}{l}\text { Smartphone app } \\
\text { for mood moni- } \\
\text { toring and dis- } \\
\text { traction }\end{array}$ & $\begin{array}{l}\text { Develop- } \\
\text { ment } \\
\text { study }\end{array}$ & $\begin{array}{l}\text { Aged 18-25 } \\
\text { years-Aus- } \\
\text { tralia }\end{array}$ & $\begin{array}{l}\text { Nonsuicidal } \\
\text { self-harm }\end{array}$ & $N / A^{g}$ & $\begin{array}{l}\text { Designed with } \\
\text { target users and } \\
\text { clinicians }\end{array}$ & $\begin{array}{l}\text { Not widely } \\
\text { available }\end{array}$ & No data \\
\hline
\end{tabular}




\begin{tabular}{|c|c|c|c|c|c|c|c|c|}
\hline $\begin{array}{l}\text { Intervention de- } \\
\text { tails }\end{array}$ & $\begin{array}{l}\text { Type of inter- } \\
\text { vention }\end{array}$ & Design & Sample & $\begin{array}{l}\text { Type of self- } \\
\text { harm }\end{array}$ & $\begin{array}{l}\text { Self-harm measure } \\
\text { used }\end{array}$ & Development & Availability & $\begin{array}{l}\text { Improve- } \\
\text { ment in } \\
\text { self-harm }\end{array}$ \\
\hline $\begin{array}{l}\text { ClinTouch } \\
\text { CareLoop en- } \\
\text { hance manage- } \\
\text { ment (2014) [39]: } \\
\text { Accessible any- } \\
\text { time; } 44 \text { daily }\end{array}$ & $\begin{array}{l}\text { Smartphone app } \\
\text { for mood moni- } \\
\text { toring and alert- } \\
\text { ing care team of } \\
\text { risk }\end{array}$ & $\begin{array}{l}\mathrm{RCT} \text { (pro- } \\
\text { tocol) }\end{array}$ & $\begin{array}{l}\text { Adults with } \\
\text { psychotic disor- } \\
\text { ders-United } \\
\text { Kingdom }\end{array}$ & $\begin{array}{l}\text { Suicidal and } \\
\text { nonsuicidal } \\
\text { self-harm }\end{array}$ & Unclear & $\begin{array}{l}\text { Codeveloped } \\
\text { with service } \\
\text { users, clinicians, } \\
\text { academics, and } \\
\text { health profession- } \\
\text { als }\end{array}$ & $\begin{array}{l}\text { Free to } \\
\text { download }\end{array}$ & No data \\
\hline
\end{tabular}

\section{ecological mo-}

mentary assess-

ment alerts

Unnamed (2016)

informative tion study

sages sent twice texts

Adults with sui-

cidal

ideation-Japan

No name (2017)
Qualita- Presented to tive study of experience

emergency department following selfharm-Canada self-harm

Cost-effective analysis (protocol)

RCT

$$
\begin{aligned}
& \text { years admitted } \\
& \text { to hospital after } \\
& \text { self-harm-Sri } \\
& \text { Lanka }
\end{aligned}
$$$$
\text { Aged 15-74 }
$$

Adults in A\&E after selfharm-Ireland

Suicidal and Unclear-selfnonsuicidal harm repetition self-harm
A-CHESS (2017)
[41]: Accessible anytime

Safe Storage In-
tervention (2015)
[42]: Texts sent
daily
Brief mobile
treatment [43]:
10 staggered
phone calls after
discharge for 24
weeks; audio
messages accessi-
ble anytime; text
reminders sent
weekly
No name (2013)
[44]: Staggered
texts that start
daily and decline
gradually to
weekly over 3
months
[45]: Accessible anytime

Smartphone app containing safety plan, social network, resources, and interaction dashboard with their therapist

Social contact via phone conversations and texts

Phone calls to monitor mood, meditation audio messages, and text reminders of treatment elements

\section{Supportive text RCT} messages

Smartphone app to provide care messages, resources, health care contacts, and self-help exercises

Unnamed (2018) Smartphone app [46]: 12-month to augment detreatment period, livery of probno further detail lem-solving therapy

TeenTEXT (2016) [47]: $R e$ Supportive texts Feasibiliwritten by the ty study ceiver sets sched- receiver ule of when texts will be received
Nonsuicidal self-harm

Researchers developed their own questionnaire measuring the presence of self-harm

Suicidal and Last question of nonsuicidal PHQ-9, hospital presentations for self-harm

Psychiatry specialists

Not widely Yes available provement of adgroups

Suicidal and Hospital presenta- No data nonsuicidal tions and coroner's self-harm data

\section{Suicidal self- Beck Scale for} harm Suicidal Ideation

No data
Developed by the network for imwith user feedback from focus

Not widely available

Not widely No data available

health care

rs on

subscription

\section{No}

available

Available for Yes

\section{RCT (pro- Adult in A\&E tocol) after self- harm-Hong \\ Suicidal and Hospital presenta- No data nonsuicidal tions and coroner's self-harm data} Kong
No data

Not widely No data available

Not widely No data available

RCT (pro- Men in A\&E af- Suicidal and Hospital presenta- No data ter selfharm-Canada nonsuicidal tions self-harm

\section{Adolescents Nonsuicidal N/A} currently self- self-harm harming-United Kingdom
Designed with Not widely No data service users with available (terminathistory of self-

harm, carers, and clinicians
Not widely No data available 


\begin{tabular}{|c|c|c|c|c|c|c|c|c|}
\hline $\begin{array}{l}\text { Intervention de- } \\
\text { tails }\end{array}$ & $\begin{array}{l}\text { Type of inter- } \\
\text { vention }\end{array}$ & Design & Sample & $\begin{array}{l}\text { Type of self- } \\
\text { harm }\end{array}$ & $\begin{array}{l}\text { Self-harm measure } \\
\text { used }\end{array}$ & Development & Availability & $\begin{array}{l}\text { Improve- } \\
\text { ment in } \\
\text { self-harm }\end{array}$ \\
\hline $\begin{array}{l}\text { Unnamed (2019) } \\
\text { [49]: Delivered } \\
\text { over 10-12 ses- } \\
\text { sions }\end{array}$ & $\begin{array}{l}\text { Audio or video } \\
\text { calls to deliver } \\
\text { problem-solv- } \\
\text { ing } \text { CBT }^{\mathrm{j}}\end{array}$ & RCT & $\begin{array}{l}\text { Aged 16-30 } \\
\text { years, depres- } \\
\text { sion and self- } \\
\text { harm-location } \\
\text { unknown }\end{array}$ & $\begin{array}{l}\text { Nonsuicidal } \\
\text { self-harm }\end{array}$ & $\begin{array}{l}\text { Urgency Persever- } \\
\text { ance Premeditation } \\
\text { Sensation-seeking } \\
\text { Impulsive Invento- } \\
\text { ry urgency sub- } \\
\text { scale }\end{array}$ & No data & $\begin{array}{l}\text { Not widely } \\
\text { available }\end{array}$ & $\begin{array}{l}\text { No data } \\
\text { (terminat- } \\
\text { ed) }\end{array}$ \\
\hline $\begin{array}{l}\text { MyPlan (2017) } \\
\text { [50]: Accessible } \\
\text { anytime }\end{array}$ & $\begin{array}{l}\text { Smartphone app } \\
\text { to store safety } \\
\text { plan }\end{array}$ & $\begin{array}{l}\text { RCT (pro- } \\
\text { tocol) }\end{array}$ & $\begin{array}{l}\text { Experiencing } \\
\text { self- } \\
\text { harm-Den- } \\
\text { mark }\end{array}$ & $\begin{array}{l}\text { Suicidal and } \\
\text { nonsuicidal } \\
\text { self-harm }\end{array}$ & $\begin{array}{l}\text { Self-reported, no } \\
\text { further information }\end{array}$ & $\begin{array}{l}\text { Developed by } \\
\text { Skovgaard } \\
\text { Larsen et al [51] }\end{array}$ & $\begin{array}{l}\text { Yes (in Den- } \\
\text { mark and } \\
\text { Norway) }\end{array}$ & No data \\
\hline $\begin{array}{l}\text { SMS SOS (2019) } \\
\text { [52]: Staggered } \\
\text { texts that start } 48 \\
h \text { after discharge } \\
\text { from A\&E, declin- } \\
\text { ing to monthly for } \\
12 \text { months }\end{array}$ & $\begin{array}{l}\text { Supportive text } \\
\text { messages }\end{array}$ & $\begin{array}{l}\text { RCT (pro- } \\
\text { tocol) }\end{array}$ & $\begin{array}{l}\text { In A\&E after } \\
\text { self- } \\
\text { harm-Aus- } \\
\text { tralia }\end{array}$ & $\begin{array}{l}\text { Suicidal and } \\
\text { nonsuicidal } \\
\text { self-harm }\end{array}$ & $\begin{array}{l}\text { Hospital presenta- } \\
\text { tions }\end{array}$ & $\begin{array}{l}\text { Developed with } \\
\text { people with lived } \\
\text { experience of } \\
\text { self-harm or } \\
\text { mental health } \\
\text { problems }\end{array}$ & $\begin{array}{l}\text { Not widely } \\
\text { available }\end{array}$ & No data \\
\hline $\begin{array}{l}\text { Crisis Text Line } \\
(2020)[53]: \text { Ac- } \\
\text { cessible anytime }\end{array}$ & $\begin{array}{l}\text { Texting with a } \\
\text { crisis counselor }\end{array}$ & $\begin{array}{l}\text { Trend } \\
\text { analysis }\end{array}$ & $\begin{array}{l}\text { No criteria-in- } \\
\text { ternational }\end{array}$ & $\begin{array}{l}\text { Suicidal and } \\
\text { nonsuicidal } \\
\text { self-harm }\end{array}$ & N/A & $\begin{array}{l}\text { Crisis text line is } \\
\text { a global organiza- } \\
\text { tion }\end{array}$ & $\begin{array}{l}\text { Available } \\
\text { globally }\end{array}$ & N/A \\
\hline $\begin{array}{l}\text { SPARX (2020) } \\
\text { [54]: Must com- } \\
\text { plete program } \\
\text { within } 6 \text { weeks }\end{array}$ & $\begin{array}{l}\text { Game-style } \\
\text { smartphone app }\end{array}$ & $\begin{array}{l}\text { RCT (pro- } \\
\text { tocol) }\end{array}$ & $\begin{array}{l}\text { Year } 8 \text { school } \\
\text { students-Aus- } \\
\text { tralia }\end{array}$ & $\begin{array}{l}\text { Suicidal and } \\
\text { nonsuicidal } \\
\text { self-harm }\end{array}$ & $\begin{array}{l}\text { Self-harm question- } \\
\text { naire }\end{array}$ & $\begin{array}{l}\text { Developed with } \\
\text { young people }\end{array}$ & $\begin{array}{l}\text { Yes (in New } \\
\text { Zealand) }\end{array}$ & No data \\
\hline $\begin{array}{l}\text { ERITA (2018) } \\
\text { [55]: } 12 \text { weeks to } \\
\text { complete } 11 \text { mod- } \\
\text { ules }\end{array}$ & $\begin{array}{l}\text { Website and } \\
\text { companion app } \\
\text { to receive treat- } \\
\text { ment for emo- } \\
\text { tion regulation }\end{array}$ & Pilot & $\begin{array}{l}\text { Adolescents } \\
\text { with self- } \\
\text { harm-Sweden }\end{array}$ & $\begin{array}{l}\text { Nonsuicidal } \\
\text { self-harm }\end{array}$ & $\begin{array}{l}\text { Deliberate self- } \\
\text { harm inventory }\end{array}$ & $\begin{array}{l}\text { Developed by the } \\
\text { authors }\end{array}$ & $\begin{array}{l}\text { Not widely } \\
\text { available }\end{array}$ & Yes \\
\hline $\begin{array}{l}\text { CATCH-IT }^{\mathrm{k}} \\
\text { (2009) [56]: Re- } \\
\text { cipients had } 3 \\
\text { phone calls, no } \\
\text { further informa- } \\
\text { tion }\end{array}$ & $\begin{array}{l}\text { Therapy provid- } \\
\text { ed via website } \\
\text { with motivation- } \\
\text { al interviewing } \\
\text { phone calls }\end{array}$ & $\mathrm{RCT}$ & $\begin{array}{l}\text { Aged 14-21 } \\
\text { years with sub- } \\
\text { threshold de- } \\
\text { pression-Unit- } \\
\text { ed States }\end{array}$ & $\begin{array}{l}\text { Nonsuicidal } \\
\text { self-harm }\end{array}$ & PHQ-A & $\begin{array}{l}\text { Developed by the } \\
\text { authors }\end{array}$ & $\begin{array}{l}\text { Not widely } \\
\text { available }\end{array}$ & Yes \\
\hline $\begin{array}{l}\text { Unnamed (1999) } \\
\text { [57]: Accessible } \\
\text { anytime for } 6 \\
\text { months following } \\
\text { discharge }\end{array}$ & $\begin{array}{l}\text { Telephone cri- } \\
\text { sis consultation } \\
\text { with on-call } \\
\text { psychiatrist }\end{array}$ & RCT & $\begin{array}{l}\text { Adult inpatients } \\
\text { with self- } \\
\text { harm-United } \\
\text { Kingdom }\end{array}$ & $\begin{array}{l}\text { Nonsuicidal } \\
\text { self-harm }\end{array}$ & $\begin{array}{l}\text { Hospital presenta- } \\
\text { tions }\end{array}$ & No data & $\begin{array}{l}\text { Not widely } \\
\text { available }\end{array}$ & No \\
\hline $\begin{array}{l}\text { Unnamed (2020) } \\
\text { [58]: A module } \\
\text { per week for } 6 \\
\text { weeks }\end{array}$ & $\begin{array}{l}\text { Web-based in- } \\
\text { tervention to } \\
\text { provide CBT- } \\
\text { based modules }\end{array}$ & RCT & $\begin{array}{l}\text { Turkish adults } \\
\text { with suicidal } \\
\text { ideation-Unit- } \\
\text { ed Kingdom } \\
\text { and Netherlands }\end{array}$ & $\begin{array}{l}\text { Suicidal self- } \\
\text { harm }\end{array}$ & $\begin{array}{l}\text { Self-harm question- } \\
\text { naire }\end{array}$ & $\begin{array}{l}\text { Developed by } \\
\text { van Spijker et al } \\
\text { [59] adapted by } \\
\text { the authors }\end{array}$ & $\begin{array}{l}\text { Not yet, but } \\
\text { will be if } \\
\text { findings are } \\
\text { positive }\end{array}$ & Yes \\
\hline $\begin{array}{l}\text { Unnamed (2018) } \\
{[60] \text { : Encouraged }} \\
\text { to write } 5 \text { mina } \\
\text { day for } 28 \text { days }\end{array}$ & $\begin{array}{l}\text { Web-based di- } \\
\text { ary }\end{array}$ & RCT & $\begin{array}{l}\text { Adults on an } \\
\text { online forum } \\
\text { who self- } \\
\text { harm-interna- } \\
\text { tional }\end{array}$ & $\begin{array}{l}\text { Suicidal and } \\
\text { nonsuicidal } \\
\text { self-harm }\end{array}$ & SITBI & $\begin{array}{l}\text { Developed by the } \\
\text { author }\end{array}$ & $\begin{array}{l}\text { Technique } \\
\text { can be adopt- } \\
\text { ed }\end{array}$ & Yes \\
\hline
\end{tabular}




\begin{tabular}{|c|c|c|c|c|c|c|c|c|}
\hline $\begin{array}{l}\text { Intervention de- } \\
\text { tails }\end{array}$ & $\begin{array}{l}\text { Type of inter- } \\
\text { vention }\end{array}$ & Design & Sample & $\begin{array}{l}\text { Type of self- } \\
\text { harm }\end{array}$ & $\begin{array}{l}\text { Self-harm measure } \\
\text { used }\end{array}$ & Development & Availability & $\begin{array}{l}\text { Improve- } \\
\text { ment in } \\
\text { self-harm }\end{array}$ \\
\hline $\begin{array}{l}\text { Uncut (2014) } \\
\text { [61]: Ecological } \\
\text { momentary as- } \\
\text { sessment }\end{array}$ & $\begin{array}{l}\text { App to track } \\
\text { mood and prac- } \\
\text { tice DBT skills, } \\
\text { therapist can } \\
\text { monitor } \\
\text { progress }\end{array}$ & $\begin{array}{l}\text { Develop- } \\
\text { ment } \\
\text { study }\end{array}$ & $\begin{array}{l}\text { Self-harm ex- } \\
\text { perts-Austria, } \\
\text { Germany, and } \\
\text { United States }\end{array}$ & $\begin{array}{l}\text { Nonsuicidal } \\
\text { self-harm }\end{array}$ & N/A & $\begin{array}{l}\text { Developed with } \\
\text { international psy- } \\
\text { chological ex- } \\
\text { perts }\end{array}$ & No data & No data \\
\hline $\begin{array}{l}\text { Living under } \\
\text { control (2017) } \\
\text { [62]: A module } \\
\text { per week for } 6 \\
\text { weeks }\end{array}$ & $\begin{array}{l}\text { Web-based in- } \\
\text { tervention pro- } \\
\text { viding } 6 \text { mod- } \\
\text { ules around } \\
\text { managing } \\
\text { thoughts and } \\
\text { feelings }\end{array}$ & $\begin{array}{l}\text { RCT (pro- } \\
\text { tocol) }\end{array}$ & $\begin{array}{l}\text { Adults-Den- } \\
\text { mark }\end{array}$ & $\begin{array}{l}\text { Suicidal and } \\
\text { nonsuicidal } \\
\text { self-harm }\end{array}$ & $\begin{array}{l}\text { Hospital records } \\
\text { and self-report } \\
\text { questionnaire (no } \\
\text { further informa- } \\
\text { tion) }\end{array}$ & $\begin{array}{l}\text { Developed by } \\
\text { van Spijker et al } \\
\text { [63] with mental } \\
\text { health profession- } \\
\text { als who work } \\
\text { with people expe- } \\
\text { riencing suicidali- } \\
\text { ty }\end{array}$ & $\begin{array}{l}\text { Not yet, but } \\
\text { will be if } \\
\text { findings are } \\
\text { positive }\end{array}$ & No data \\
\hline $\begin{array}{l}\text { Crisis Care } \\
\text { (2017) [64]: Ac- } \\
\text { cessible anytime }\end{array}$ & $\begin{array}{l}\text { Smartphone app } \\
\text { containing cop- } \\
\text { ing skills, dis- } \\
\text { traction activi- } \\
\text { ties, and a help } \\
\text { me now section }\end{array}$ & $\begin{array}{l}\text { Pilot } \\
\text { study }\end{array}$ & $\begin{array}{l}\text { Adolescent psy- } \\
\text { chiatry outpa- } \\
\text { tients-United } \\
\text { States }\end{array}$ & $\begin{array}{l}\text { Suicidal self- } \\
\text { harm }\end{array}$ & N/A & $\begin{array}{l}\text { Developed by the } \\
\text { author }\end{array}$ & $\begin{array}{l}\text { Not widely } \\
\text { available }\end{array}$ & Yes \\
\hline $\begin{array}{l}\text { Monsenso (2020) } \\
\text { [65]: Accessible } \\
\text { anytime }\end{array}$ & $\begin{array}{l}\text { Smartphone app } \\
\text { providing DBT } \\
\text { skills and mood } \\
\text { monitoring; } \\
\text { links with clini- } \\
\text { cians database }\end{array}$ & $\begin{array}{l}\mathrm{RCT} \text { (pro- } \\
\text { tocol) }\end{array}$ & $\begin{array}{l}\text { Adults with } \\
\text { borderline per- } \\
\text { sonality disor- } \\
\text { der and self- } \\
\text { harm-Den- } \\
\text { mark }\end{array}$ & $\begin{array}{l}\text { Suicidal and } \\
\text { nonsuicidal } \\
\text { self-harm }\end{array}$ & $\begin{array}{l}\text { Self-harm invento- } \\
\text { ry }\end{array}$ & $\begin{array}{l}\text { Developed by the } \\
\text { author }\end{array}$ & $\begin{array}{l}\text { Available for } \\
\text { health care } \\
\text { providers on } \\
\text { subscription }\end{array}$ & No data \\
\hline $\begin{array}{l}\text { Unnamed (2019) } \\
\text { [66]: No details } \\
\text { yet }\end{array}$ & $\begin{array}{l}\text { Supportive text } \\
\text { messages }\end{array}$ & $\begin{array}{l}\text { Develop- } \\
\text { ment } \\
\text { study }\end{array}$ & $\begin{array}{l}\text { Adolescents } \\
\text { with self- } \\
\text { harm-China }\end{array}$ & $\begin{array}{l}\text { Nonsuicidal } \\
\text { self-harm }\end{array}$ & N/A & $\begin{array}{l}\text { Developed with } \\
\text { youth with lived } \\
\text { experience of } \\
\text { self-harm }\end{array}$ & No data & No data \\
\hline $\begin{array}{l}\text { Unnamed (2020) } \\
\text { [67]: Unlimited } \\
\text { access every } \\
\text { evening }\end{array}$ & $\begin{array}{l}\text { Peer-supported } \\
\text { hotline }\end{array}$ & $\begin{array}{l}\text { Trend } \\
\text { analysis }\end{array}$ & $\begin{array}{l}\text { Teenagers-Unit- } \\
\text { ed States }\end{array}$ & $\begin{array}{l}\text { Suicidal and } \\
\text { nonsuicidal } \\
\text { self-harm }\end{array}$ & N/A & $\begin{array}{l}\text { Founded by men- } \\
\text { tal health profes- } \\
\text { sionals }\end{array}$ & $\begin{array}{l}\text { Available to } \\
\text { teenagers in } \\
\text { the United } \\
\text { States }\end{array}$ & N/A \\
\hline
\end{tabular}

${ }^{\mathrm{a}}$ Italics refer to the intervention duration.

${ }^{\mathrm{b}} \mathrm{RCT}$ : randomized controlled trial.

${ }^{\mathrm{c}} \mathrm{A} \& \mathrm{E}$ : accident \& emergency.

${ }^{\mathrm{d}}$ EMA: ecological momentary assessment.

ePHQ: patient health questionnaire.

${ }^{\mathrm{f}} \mathrm{CAMHS}$ : Child and adolescent mental health services.

gN/A: not applicable.

${ }^{h}$ DBT: dialectical behavior therapy.

${ }^{\mathrm{i}}$ SITBI: self-injurious thoughts and behaviors interview.

${ }^{\mathrm{j}} \mathrm{CBT}$ : cognitive behavioral therapy.

${ }^{\mathrm{k}}$ CATCH-IT: Competent Adulthood Transition with Cognitive Behavioural Humanistic and Interpersonal Training.

The 36 papers related to 35 separate studies, 2 of which were published from the same study with one paper detailing the quantitative findings [36] and the other the qualitative findings [37]. They were therefore not removed as duplicates; however, as the study details were the same, the study characteristics were merged to not present the same information twice.

A total of 12 papers reported protocols. The corresponding authors were contacted to request any update or preliminary findings; a preprint paper was received from one, so the protocol was replaced with this and the data were extracted. The remaining 11 protocols had no further data at the time of this review (April-June 2020).

The most common study design was the RCT (20/35, 57\%), with most papers published between 2014 and 2020 (33/35, $94 \%)$. Studies were most commonly conducted in Europe $(14 / 35,40 \%)$ and North America $(7 / 35,20 \%)$. Most studies measured self-harm using various self-report questionnaires $(18 / 35,51 \%)$ and looked at both suicidal and nonsuicidal self-harm $(19 / 35,54 \%)$. 


\section{Participants}

The participant data are summarized in Table 2. Sample sizes ranged from 3 to 122,909 , involving mostly clinical cohorts $(24 / 35,69 \%)$, and approximately half were adult samples $(18 / 35$, $51 \%$ ), with one focusing specifically on young adults (aged $18-25$ years). None of the studies included children aged $<12$ years and adults aged $>65$ years. Gender was reported in 18 papers, with the majority including more females than males $(14 / 18,78 \%)$; only 1 study included a nonbinary participant $(1 / 18,6 \%)$. Most papers provided no data on ethnicity $(25 / 35$, $71 \%)$ and those that did recruited mostly White people $(8 / 10$, $80 \%)$. Presenting problems screened for or required as inclusion criteria among the samples were primarily self-harm $(26 / 35$, $74 \%)$, depression $(22 / 35,63 \%)$, suicidal ideation $(15 / 35,43 \%)$, and suicide attempts $(15 / 35,43 \%)$.

Table 2. Participant characteristics $(n=35){ }^{\mathrm{a}}$

\begin{tabular}{|c|c|c|}
\hline Characteristic & $\mathrm{n}(\%)$ & Study \\
\hline \multicolumn{3}{|l|}{ Age (years) } \\
\hline Adults & $18(54)$ & {$[30-32,35,38-41,44-46,48,57,58,60-62,65]$} \\
\hline Adolescents (up to 18 years) & $8(23)$ & {$[33,36,37,47,54,55,64,66,67]$} \\
\hline Adolescents and adults & $6(17)$ & {$[29,34,43,49,52,56]$} \\
\hline No age restriction & $1(3)$ & {$[50]$} \\
\hline Unknown & $2(5)$ & {$[42,53]$} \\
\hline \multicolumn{3}{|l|}{ Population } \\
\hline Clinical & $24(69)$ & {$[29,30,32,33,36-41,43-50,52,55-57,64-66]$} \\
\hline Community & $9(26)$ & {$[31,35,42,53,54,58,60,62,67]$} \\
\hline Clinical and community & $1(3)$ & {$[34]$} \\
\hline Mental health professionals & $1(3)$ & {$[61]$} \\
\hline \multicolumn{3}{|c|}{ Presenting problems or inclusion criteria } \\
\hline Self-harm & $26(74)$ & {$[29,30,34-37,40,41,43-50,52,53,55-58,60,62,65-67]$} \\
\hline Depression & $22(63)$ & {$[29,31-34,36-38,40,41,43,45,48-50,53-56,60,62,66,67]$} \\
\hline Anxiety & $13(37)$ & {$[29,31,32,34,36,37,41,48,49,53-55,66,67]$} \\
\hline Suicidal ideation & $15(43)$ & {$[29,30,32,34,35,40,43-45,50,53,58,60,62,64]$} \\
\hline Suicide attempt & $15(43)$ & {$[29,30,32,34,35,44-46,48,50,53,57,60,64,65]$} \\
\hline Borderline personality disorder & $5(14)$ & {$[31,32,48,55,65]$} \\
\hline Psychosis & $4(11)$ & {$[32,39,40,66]$} \\
\hline Substance or alcohol use & $4(11)$ & {$[32,41,43,57]$} \\
\hline Eating disorders & $2(6)$ & {$[31,55]$} \\
\hline Sleep disorders & $2(6)$ & {$[32,54]$} \\
\hline Neurological disorders & $1(3)$ & {$[32]$} \\
\hline Somatoform disorder & $1(3)$ & {$[32]$} \\
\hline
\end{tabular}

${ }^{\mathrm{a}}$ Grist et al (2018) [36] and Stallard et al (2018) [37] are separate papers from the same study.

Understand the Types and Features of Interventions That Have Been Developed and Evaluated

\section{Characteristics of mHealth Interventions}

Intervention characteristics are summarized in Table 3 and relate to 34 interventions. As mentioned earlier, another 2 papers reported on different trials of the same intervention. Most studies described apps $(15 / 34,44 \%)$ or texting or calling services $(13 / 34,38 \%)$, and most interventions required a mobile phone $(16 / 34,47 \%)$ or a smartphone $(11 / 34,32 \%)$, whereas the rest required any internet-enabled device $(4 / 34,12 \%)$ or an iPod touch $(1 / 34,3 \%)$. Approximately half of the interventions did not include any face-to-face support $(16 / 34,47 \%)$; of these, 9 were standalone interventions $(9 / 16,56 \%), 7$ included an element of human support provided digitally (ie, texts from a clinician; 7/16, 21\%), and 2 exclusively provided digital support (ie, a hotline; $2 / 16,13 \%$ ). A range of underpinning therapeutic models informing the intervention content were reported, with 21 studies $(21 / 34,62 \%)$ citing at least one approach and cognitive behavioral therapy being the most common (10/34, $29 \%$ ). Supportive messages or phone calls were the most common elements among the interventions $(14 / 34,41 \%)$. Only $3(9 \%)$ papers specified that the interventions contained a safety plan. 
Table 3. Intervention characteristics $(n=34){ }^{a}$

\begin{tabular}{|c|c|c|}
\hline Intervention characteristics & $\mathrm{n}(\%)$ & Study \\
\hline \multicolumn{3}{|l|}{ Intervention type } \\
\hline Apps & $15(44)$ & {$[31,32,34,36,38,39,41,45,48,50,54,61,64,65]$} \\
\hline Texting or calling services & $13(38)$ & {$[30,33,40,42-44,47,49,52,53,57,66,67]$} \\
\hline Websites or web-based therapies & $4(12)$ & {$[55,56,58,62]$} \\
\hline Web-based diary & $1(3)$ & {$[60]$} \\
\hline Social network & $1(3)$ & [29] \\
\hline \multicolumn{3}{|l|}{ Device } \\
\hline Mobile phone & $16(47)$ & {$[30,33,39,40,42-45,47,49,52,53,57,65-67]$} \\
\hline Smartphone & $11(32)$ & {$[31,32,34-38,41,46,50,54,55,60,61,64]$} \\
\hline Any internet-enabled device & $4(12)$ & {$[29,56,58,62]$} \\
\hline iPod touch (or iPhone) & $1(3)$ & [48] \\
\hline \multicolumn{3}{|l|}{ Human support included } \\
\hline Face-to-face provided & $12(35)$ & {$[31,32,34,36-38,41,43,46-48,50,52,65]$} \\
\hline Digital support provided & $7(21)$ & {$[29,49,53,55,57,58,67]$} \\
\hline No support & $9(27)$ & {$[30,33,35,44,54,60,62,64,66]$} \\
\hline Both compared & $2(6)$ & {$[45,56]$} \\
\hline Not specified & $4(12)$ & {$[39,40,42,61]$} \\
\hline \multicolumn{3}{|l|}{ Underpinning therapeutic model } \\
\hline Cognitive behavioral therapy & $10(29)$ & {$[31,32,36-38,47,49,54,56,58,62,64]$} \\
\hline Dialectical behavior therapy & $6(18)$ & {$[31,32,36-38,48,61,65]$} \\
\hline Cognitive & $3(9)$ & {$[29,34,50]$} \\
\hline Behavioral & $3(9)$ & {$[35-37,56]$} \\
\hline Mindfulness & $2(6)$ & {$[29,36,37]$} \\
\hline Problem-solving therapy & $2(6)$ & {$[41,46]$} \\
\hline Acceptance and commitment therapy & $1(3)$ & {$[56]$} \\
\hline Interpersonal psychotherapy & $1(3)$ & {$[56]$} \\
\hline Autobiographic self enhancement & $1(3)$ & {$[60]$} \\
\hline Not specified & $13(38)$ & {$[30,33,39,40,42-45,52,53,57,66,67]$} \\
\hline \multicolumn{3}{|l|}{ Features } \\
\hline Supportive messages or phone calls & $14(41)$ & {$[30,33,34,40,42-45,47,52,53,57,66,67]$} \\
\hline Coping skills & $9(27)$ & {$[40,48,54,56,58,61,62,64,65]$} \\
\hline Mood diaries & $8(24)$ & {$[34,36-39,45,60,61,65]$} \\
\hline Links to helplines, services, or caregivers & $8(24)$ & {$[33,40,41,44-46,53,64]$} \\
\hline Problem-solving techniques & $6(18)$ & {$[29,41,43,46,54,56]$} \\
\hline Alerts to clinicians & $6(18)$ & {$[33,39,41,47,61,65]$} \\
\hline Mood lifting or physical activities & $6(18)$ & {$[34,36,37,44,54,56,64]$} \\
\hline Information and psychoeducation & $5(15)$ & {$[29,41,44,54,65]$} \\
\hline Relaxation and meditation & $5(15)$ & {$[31,32,36,38,45,64]$} \\
\hline Mindfulness & $5(15)$ & {$[29,31,32,36,37,48,58]$} \\
\hline Thought challenging & $5(15)$ & {$[36,37,54,56,58,62]$} \\
\hline Photos, music, and other media & $4(12)$ & {$[31,32,34,36,37,41]$} \\
\hline Medication and intervention reminders & $4(12)$ & {$[34,39,40,43]$} \\
\hline
\end{tabular}




\begin{tabular}{lll}
\hline Intervention characteristics & $\mathrm{n}(\%)$ & Study \\
\hline Social and peer support & $3(9)$ & {$[29,41,67]$} \\
Safety plan & $3(9)$ & {$[41,50,62]$} \\
Distraction methods & $3(9)$ & {$[31,32,38,64]$} \\
Games & $3(9)$ & {$[31,35,54]$} \\
\hline
\end{tabular}

${ }^{\mathrm{a}}$ Bush et al (2015) [31] and Bush et al (2017) [32] relate to the same intervention; Grist et al (2018) [36] and Stallard et al (2018) [37] are separate papers from the same study.

\section{Development and Availability}

Half of the mHealth interventions were developed with multiple collaborators $(17 / 34,50 \%)$, including mental health professionals $(10 / 34,29 \%)$, target end users $(11 / 34,32 \%)$, and companies or organizations $(3 / 34,9 \%)$. Most interventions were not currently available to the public $(20 / 34,59 \%)$. Conversely, only $15 \%(5 / 34)$ are publicly available, whereas others are available for purchase by health care professionals or services $(4 / 34,12 \%)$ or users $(1 / 34,3 \%)$. Table 1 provides further details on the intervention development and availability.

\section{Highlight Research Findings Around mHealth Interventions for Self-Harm}

\section{Study Findings}

Of the 19 papers that reported outcomes, 14 (74\%) reported positive findings postintervention. Of the 5 studies that did not report positive findings, $2(26 \%)$ were terminated during recruitment due to a lack of feasibility. One study noted that this was due to high levels of depression and the reluctance of participants who self-harmed to engage with mental health services [49], whereas the other suggested it was a good intervention but Child and Adolescent Mental Health Services was the wrong setting due to clinicians' time constraints [47]. Furthermore, 3 studies reported no significant effect of the intervention on self-harm recovery [32,43,57], one of which cited past episodes of self-harm as a barrier to efficacy [57].
Clinician and parental attitudes were typically favorable toward the interventions $[31,32,38,41,47,64]$, with only 2 papers reporting concerns $[38,47]$. The identified benefits include promoting self-efficacy $[32,58]$, helping difficult disclosure [33], immediate access [43], time and cost benefits [55], encouraging help-seeking [40], being useful in crises [64,66], and having a positive influence on the therapeutic alliance in the face-to-face element within blended approaches [41,58]. Barriers were not commonly mentioned in the papers, although 2 papers did note that digital interventions that were administered by a mental health worker posed challenges due to the lack of engagement with mental health services among people who self-harm $[47,49]$.

\section{Determine What Outcomes Are Typically Used to Assess the Efficacy of Interventions}

The study outcomes are summarized in Table 4. Most studies had multiple outcomes related to self-harm $(21 / 35,60 \%)$, suicide attempts $(19 / 35,54 \%)$, suicidal ideation $(16 / 35,46 \%)$, intervention experience $(16 / 35,46 \%)$, and engagement with the intervention $(13 / 35,37 \%)$. Other mental health issues such as depression $(16 / 35,46 \%)$ and anxiety $(9 / 35,26 \%)$ were also considered. Outcomes related to self-harm mostly focused on episode frequency $(14 / 21,67 \%)$, whereas others focused on repeated presentations to hospital $(5 / 21,24 \%)$ or self-harm thoughts or urges $(4 / 21,19 \%)$. 
Table 4. Study outcomes $(n=35){ }^{a}$

\begin{tabular}{|c|c|c|}
\hline Outcome & $\mathrm{n}(\%)$ & Study \\
\hline \multicolumn{3}{|l|}{ Mental health } \\
\hline $\mathrm{SH}^{\mathrm{b}}$ frequency & $14(40)$ & {$[29,34-37,39,40,42,44,45,54-60,62]$} \\
\hline Presentations to hospital for SH & $5(14)$ & {$[40,45,46,52,57]$} \\
\hline SH thoughts or urges & $4(11)$ & {$[34,48,56,60]$} \\
\hline SH (specifics unclear) & $2(6)$ & {$[50,58]$} \\
\hline Suicidal ideation & $16(46)$ & {$[29,30,32,34,35,40,43-45,49,50,54,58,60,62,65]$} \\
\hline Suicide attempts & $19(54)$ & {$[29,30,32,34,35,40,42,43,45,46,48-50,52,57,58,60,62,65]$} \\
\hline Depression & $16(46)$ & {$[29,30,34,36-38,43,45,49,50,53,54,56,58,60,62,66]$} \\
\hline Anxiety & $9(26)$ & {$[29,30,34,36,37,49,53,54,58,62]$} \\
\hline Eating disorders & $3(9)$ & {$[30,53,54]$} \\
\hline Borderline personality disorder & $2(6)$ & {$[55,65]$} \\
\hline Psychosis & $1(3)$ & {$[54]$} \\
\hline \multicolumn{3}{|l|}{ Other well-being } \\
\hline Other mental well-being & $7(20)$ & {$[29,48,53-55,60,62]$} \\
\hline Interpersonal issues & $7(20)$ & {$[29,32,43,45,49,53,54]$} \\
\hline Hopelessness & $6(17)$ & {$[44,45,49,50,58,62]$} \\
\hline Sleep & $3(9)$ & {$[29,33,54]$} \\
\hline Alcohol or substance use & $3(9)$ & {$[43,53,54]$} \\
\hline Quality of life & $3(9)$ & {$[29,58,62]$} \\
\hline Other self-destructive behaviors & $2(6)$ & {$[54,55]$} \\
\hline \multicolumn{3}{|c|}{ Intervention feasibility and acceptability } \\
\hline Experience & $16(46)$ & {$[29-34,36,37,44,47,48,50,54,58,60,62,64,66]$} \\
\hline Engagement & $13(37)$ & {$[29,31-34,39,44,45,48,53,54,56,67]$} \\
\hline Health care costs & $1(3)$ & [46] \\
\hline
\end{tabular}

${ }^{a}$ Grist et al (2018) [36] and Stallard et al (2018) [37] are separate papers from the same study.

${ }^{\mathrm{b}} \mathrm{SH}$ : self-harm.

\section{Discussion}

\section{Principal Findings}

This scoping review identified 36 papers relating to 34 separate mHealth tools for managing self-harm. Papers were primarily RCTs and protocols published in Europe or North America within the last 6 years. This recent increase in papers reflects the growing interest in developing evidence-based mHealth interventions to improve access to psychological therapies. The large number of protocols suggests that this trend will continue as more findings are published in the coming years. Participants were mostly White adult females recruited from clinical populations, with only one nonbinary participant across all the studies, and many did not report the ethnicity of their participants. This is concerning given the high prevalence of self-harm found in both ethnic [68] and gender minorities [69]. It is possible that other nonbinary or gender-diverse individuals participated in these studies, but they were not truly represented in the way that the researchers assessed or reported participant demographics.
Depression and anxiety were the 2 most highly studied comorbidities, which is consistent with research suggesting strong correlations between these disorders and self-harm [70]. The interventions most commonly studied were not blended with any face-to-face support and were mostly text or call-based services or apps used on mobile phones or smartphones. Considering the ubiquity of both mobile phones [17,71] and smartphones [71], this is a positive finding and suggests that there are indeed mHealth interventions that could be more widely accessed. Interestingly, no interventions designed for use on other devices, such as wearable technologies, have been identified. This is despite research suggesting that they are acceptable for treating mental health issues among those who do not typically engage with mental health services [72]. However, this is still a relatively new area, and wearable devices designed to treat or help manage mental health difficulties are predicted to increase over the next few years [73].

Several studies did not specify any underpinning therapeutic models informing the content of their intervention, which raises concerns given the unhelpful and even dangerous advice that 
has been found in other freely available mental health apps [74]. Similarly, less than a third of the interventions were developed with individuals who have lived experience of self-harm, despite evidence suggesting that this could lead to more effective interventions being developed. Given the expertise of those with lived experience of self-harm, their input is essential $[75,76]$.

Most studies testing interventions reported overall promising findings, suggesting that mHealth can be a viable tool for people struggling with self-harm. The study outcomes largely promoted a reduction in self-harm frequency over and above others, such as a reduction in self-harm urges or severity. Although reduced frequency is a common measure of self-harm recovery within research, it has been argued that it is not advisable to rely solely on this; a reduction in self-harm episodes may mean that each episode has become more severe or has been replaced with a different type of self-destructive behavior [77]. Overall, there needs to be a more consistent framework to assess outcomes from self-harm research, with further research looking into the maintenance of positive outcomes. Similarly, there was considerable variation in the tools used to measure self-harm across the studies. This makes comparisons between studies difficult; therefore, greater consistency may also be beneficial.

Another commonly observed finding was the clinicians' favorable attitudes toward the intervention. This reflects overall positive attitudes and an eagerness to incorporate technology into practice found in research on clinicians' attitudes toward technology in mental health care [78]. This is important considering that there is research highlighting that clinicians' attitudes are pivotal in intervention implementation [79].

A barrier to the implementation of blended interventions that required clinician involvement was the lack of engagement that people who self-harm have with mental health services. This is consistent with the body of literature that corroborates the lack of professional help-seeking among people who self-harm [8-11] and further emphasizes the need for mHealth interventions for self-harm that individuals can access easily and discretely. Despite many studies having positive outcomes, it seems that not many of the interventions studied are readily available to the general public or even to those attending mental health services. Moreover, few papers made this information apparent, so information on availability was sought from internet and app store searches and by contacting the authors of the papers.

\section{Implications for Future Research}

This review identified several limitations in the current literature on mHealth tools for managing self-harm. Notably, research thus far has been limited to White adult females from western societies, yet self-harm has been identified as a significant issue among minority groups [68,69], highlighting the need to diversify research by recruiting understudied groups such as males and minority populations who may also benefit from mHealth interventions. It is also important for future studies to report the ethnicity of participants. Similarly, participants have mostly been from clinical samples; however, given that people who self-harm do not often seek professional help, it is possible that findings from clinical samples may not necessarily be generalizable to wider populations who self-harm. Therefore, it is important to assess the efficacy of interventions in community samples. It is also essential for more intervention developers to collaborate with people who have lived experience of self-harm, given the limited instances thus far highlighted in this review.

Another notable point is that the measurements of self-harm recovery were typically restricted to a reduction in the frequency of episodes and, although this may be a useful assessment, it may also be worth considering measuring other elements as well, such as the severity of self-harming episodes or any other substitute self-destructive behaviors.

Another consideration to take forward from this review is the reliance on RCTs to evaluate digital interventions. Although RCTs have long been considered the gold standard method, the pace at which digital interventions develop and evolve means that the data can be outdated before the trial has been completed [80-82]. Following this, it may be prudent for future research to also consider different study designs, such as pre- and posttests that can keep up with the rapid development of digital interventions. Furthermore, RCTs can indicate whether an intervention is effective but may be limited in their ability to explore the reason [83]. Therefore, it may also be beneficial for future research to apply the NIMH's experimental therapeutics approach or use qualitative studies that can contribute further to the understanding of how and why certain interventions are effective as well as whether they are safe and do no harm. Similarly, the application of the experimental therapeutic approach may contribute further to the understanding of how and why certain interventions are effective [84]. Future work should also focus on the implementation and dissemination of effective interventions, following the lack of availability of interventions within the papers in this review.

\section{Limitations}

As the purpose of this review was to collate the data available on mHealth tools for managing self-harm, there was no scope to conduct a quality assessment of the included studies. This means that we cannot verify that the research included here is of sufficient quality to draw concrete inferences from. This review may also have been limited by the decision to not include any papers for which there was no English version available. Nonetheless, this review offers insight into the current evidence base for mHealth interventions for self-harm.

\section{Conclusions}

This review has synthesized the current evidence for mHealth tools for managing self-harm. Overall, there are useful interventions that have been developed to promote recovery from self-harm. However, certain limitations pose challenges in drawing firm conclusions from the included studies. Suggestions for how future research can improve upon this have been made, in the hope of developing a robust evidence base so that clinicians and users are better equipped to make informed decisions about which mHealth tools to use. This will hopefully help to overcome some of the barriers that people who self-harm face in accessing support. 


\section{Conflicts of Interest}

None declared.

\section{Multimedia Appendix 1}

Full search strategy.

[DOC File, 28 KB-Multimedia Appendix 1]

\section{References}

1. Self-Harm. National Institute for Health and Care Excellence. 2013. URL: https://www.nice.org.uk/guidance/qs34 [accessed 2020-08-17]

2. Lundh L, Wångby-Lundh M, Bjärehed J. Deliberate self-harm and psychological problems in young adolescents: evidence of a bidirectional relationship in girls. Scand J Psychol 2011 Oct;52(5):476-483. [doi: 10.1111/j.1467-9450.2011.00894.x] [Medline: 21585393]

3. Tsiachristas A, McDaid D, Casey D, Brand F, Leal J, Park A, et al. General hospital costs in England of medical and psychiatric care for patients who self-harm: a retrospective analysis. Lancet Psych 2017 Oct;4(10):759-767 [FREE Full text] [doi: 10.1016/S2215-0366(17)30367-X] [Medline: 28890321]

4. Mars B, Heron J, Klonsky ED, Moran P, O'Connor RC, Tilling K, et al. Predictors of future suicide attempt among adolescents with suicidal thoughts or non-suicidal self-harm: a population-based birth cohort study. Lancet Psychiatry 2019 Apr;6(4):327-337 [FREE Full text] [doi: 10.1016/S2215-0366(19)30030-6] [Medline: 30879972]

5. McManus S, Gunnell D, Cooper C, Bebbington PE, Howard LM, Brugha T, et al. Prevalence of non-suicidal self-harm and service contact in England, 2000-14: repeated cross-sectional surveys of the general population. Lancet Psychiatry 2019 Jul;6(7):573-581 [FREE Full text] [doi: 10.1016/S2215-0366(19)30188-9] [Medline: $\underline{31175059]}$

6. Gratz KL. Risk Factors for and Functions of Deliberate Self - Harm: An Empirical and Conceptual Review. Clinical Psychology: Science and Practice 2006 May 11;10(2):192-205. [doi: 10.1093/clipsy.bpg022]

7. Whitlock J, Eckenrode J, Silverman D. Self-injurious behaviors in a college population. Pediatrics 2006 Jun;117(6):1939-1948. [doi: 10.1542/peds.2005-2543] [Medline: 16740834]

8. Tørmoen AJ, Rossow I, Mork E, Mehlum L. Contact with child and adolescent psychiatric services among self-harming and suicidal adolescents in the general population: a cross sectional study. Child Adolesc Psychiatry Ment Health 2014;8:13 [FREE Full text] [doi: 10.1186/1753-2000-8-13] [Medline: 24742154]

9. Michelmore L, Hindley P. Help-seeking for suicidal thoughts and self-harm in young people: a systematic review. Suicide Life Threat Behav 2012 Oct;42(5):507-524. [doi: 10.1111/j.1943-278X.2012.00108.x] [Medline: 22889130]

10. Nada-Raja S, Morrison D, Skegg K. A population-based study of help-seeking for self-harm in young adults. Aust N Z J Psychiatry 2003 Oct;37(5):600-605. [Medline: 14511089 ]

11. Fortune S, Sinclair J, Hawton K. Help-seeking before and after episodes of self-harm: a descriptive study in school pupils in England. BMC Public Health 2008 Oct 24;8:369 [FREE Full text] [doi: 10.1186/1471-2458-8-369] [Medline: 18947435]

12. NICE. Self-harm in over 8s: long-term management. National Institute for Health and Care Excellence. 2011 Nov 23. URL: https://www.nice.org.uk/guidance/cg133 [accessed 2020-05-15]

13. Hawton K, Witt KG, Taylor Salisbury TL, Arensman E, Gunnell D, Hazell P, et al. Pharmacological interventions for self-harm in adults. Cochrane Database Syst Rev 2015 Jul 06(7):CD011777. [doi: 10.1002/14651858.CD011777] [Medline: 26147958]

14. Hawton K, Witt KG, Taylor STL, Arensman E, Gunnell D, Hazell P, et al. Psychosocial interventions for self-harm in adults. Cochrane Database Syst Rev 2016 May 12(5):CD012189. [doi: 10.1002/14651858.CD012189] [Medline: 27168519]

15. Cummins I. The Impact of Austerity on Mental Health Service Provision: A UK Perspective. Int J Environ Res Public Health 2018 Jun 01;15(6):1145-1145 [FREE Full text] [doi: 10.3390/ijerph15061145] [Medline: 29865157]

16. World HO. New horizons for health through mobile technologies. Geneva: World Health Organization; 2011.

17. Ofcom. Children and parents: Media use and attitudes report 2018. Ofcom. 2019. URL: https://www.ofcom.org.uk/ data/ assets/pdf file/0024/134907/children-and-parents-media-use-and-attitudes-2018.pdf [accessed 2020-01-27]

18. Ofcom. Adults: Media use and attitudes report 2019. Ofcom. 2019. URL: https://www.ofcom.org.uk/ data/assets/pdf file/ 0021/149124/adults-media-use-and-attitudes-report.pdf [accessed 2020-03-27]

19. Barak A, Klein B, Proudfoot JG. Defining internet-supported therapeutic interventions. Ann Behav Med 2009 Aug;38(1):4-17. [doi: 10.1007/s12160-009-9130-7] [Medline: 19787305]

20. Clough BA, Casey LM. The smart therapist: A look to the future of smartphones and mHealth technologies in psychotherapy. Professional Psychology: Research and Practice 2015;46(3):147-153. [doi: 10.1037/pro0000011]

21. Firth J, Torous J, Nicholas J, Carney R, Rosenbaum S, Sarris J. Can smartphone mental health interventions reduce symptoms of anxiety? A meta-analysis of randomized controlled trials. J Affect Disord 2017 Aug 15;218:15-22 [FREE Full text] [doi: 10.1016/j.jad.2017.04.046] [Medline: 28456072]

22. Firth J, Torous J. Smartphone Apps for Schizophrenia: A Systematic Review. JMIR Mhealth Uhealth 2015;3(4):e102 [FREE Full text] [doi: 10.2196/mhealth.4930] [Medline: 26546039] 
23. Arean PA, Hallgren KA, Jordan JT, Gazzaley A, Atkins DC, Heagerty PJ, et al. The Use and Effectiveness of Mobile Apps for Depression: Results From a Fully Remote Clinical Trial. J Med Internet Res 2016 Dec 20;18(12):e330 [REEE Full text] [doi: 10.2196/jmir.6482] [Medline: 27998876]

24. Prada P, Zamberg I, Bouillault G, Jimenez N, Zimmermann J, Hasler R, et al. EMOTEO: A Smartphone Application for Monitoring and Reducing Aversive Tension in Borderline Personality Disorder Patients, a Pilot Study. Perspect Psychiatr Care 2017 Oct;53(4):289-298. [doi: 10.1111/ppc.12178] [Medline: 27439663]

25. Witt K, Spittal MJ, Carter G, Pirkis J, Hetrick S, Currier D, et al. Effectiveness of online and mobile telephone applications ('apps') for the self-management of suicidal ideation and self-harm: a systematic review and meta-analysis. BMC Psychiatry 2017 Aug 15;17(1):297 [FREE Full text] [doi: 10.1186/s12888-017-1458-0] [Medline: 28810841]

26. Melia R, Francis K, Hickey E, Bogue J, Duggan J, O'Sullivan M, et al. Mobile Health Technology Interventions for Suicide Prevention: Systematic Review. JMIR Mhealth Uhealth 2020 Jan 15;8(1):e12516. [doi: 10.2196/12516]

27. Arshad U, Farhat-Ul-Ain, Gauntlett J, Husain N, Chaudhry N, Taylor PJ. A Systematic Review of the Evidence Supporting Mobile- and Internet-Based Psychological Interventions For Self-Harm. Suicide Life Threat Behav 2020 Feb;50(1):151-179 [FREE Full text] [doi: 10.1111/sltb.12583] [Medline: 31448847]

28. Cliffe B. What mHealth interventions are available for individuals who are struggling with self-harm? Open Science Framework. 2020. URL: https://osf.io/ay5pf/

29. Cochrane. Preventing relapse of major depressive disorder in youth: randomised Controlled Trial of a novel mindfulness-based cognitive online social therapy. Cochrane. 2019. URL: https://www.cochranelibrary.com/central/doi/10.1002/central/ CN-02064859/full [accessed 2020-04-23]

30. Berrouiguet S, Alavi Z, Vaiva G, Courtet P, Baca-García E, Vidailhet P, et al. SIAM (Suicide intervention assisted by messages): the development of a post-acute crisis text messaging outreach for suicide prevention. BMC Psychiatry 2014;14:294 [FREE Full text] [doi: 10.1186/s12888-014-0294-8] [Medline: 25404215]

31. Bush NE, Dobscha SK, Crumpton R, Denneson LM, Hoffman JE, Crain A, et al. A Virtual Hope Box smartphone app as an accessory to therapy: proof-of-concept in a clinical sample of veterans. Suicide Life Threat Behav 2015 Feb;45(1):1-9. [doi: 10.1111/sltb.12103] [Medline: 24828126]

32. Bush NE, Smolenski DJ, Denneson LM, Williams HB, Thomas EK, Dobscha SK. A Virtual Hope Box: Randomized Controlled Trial of a Smartphone App for Emotional Regulation and Coping With Distress. Psychiatr Serv 2017 Apr 01;68(4):330-336. [doi: 10.1176/appi.ps.201600283] [Medline: 27842473]

33. Chen RY, Feltes JR, Tzeng WS, Lu ZY, Pan M, Zhao N, et al. Phone-Based Interventions in Adolescent Psychiatry: A Perspective and Proof of Concept Pilot Study With a Focus on Depression and Autism. JMIR Res Protoc 2017 Jun 16;6(6):e114 [FREE Full text] [doi: 10.2196/resprot.7245] [Medline: 28623183]

34. Di Simplicio M, Appiah-Kusi E, Wilkinson P, Watson P, Meiser-Stedman C, Kavanagh DJ, et al. Imaginator: A Proof-of-Concept Feasibility Trial of a Brief Imagery-Based Psychological Intervention for Young People Who Self-Harm. Suicide Life Threat Behav 2020 Jun;50(3):724-740. [doi: 10.1111/sltb.12620] [Medline: 32057131]

35. Franklin JC, Fox KR, Franklin CR, Kleiman EM, Ribeiro JD, Jaroszewski AC, et al. A brief mobile app reduces nonsuicidal and suicidal self-injury: Evidence from three randomized controlled trials. J Consult Clin Psychol 2016 Jun;84(6):544-557. [doi: 10.1037/ccp0000093] [Medline: 27018530]

36. Grist R, Porter J, Stallard P. Acceptability, Use, and Safety of a Mobile Phone App (BlueIce) for Young People Who Self-Harm: Qualitative Study of Service Users' Experience. JMIR Ment Health 2018 Feb 23;5(1):e16 [FREE Full text] [doi: 10.2196/mental.8779] [Medline: 29475823]

37. Stallard P, Porter J, Grist R. A Smartphone App (BlueIce) for Young People Who Self-Harm: Open Phase 1 Pre-Post Trial. JMIR Mhealth Uhealth 2018 Jan 30;6(1):e32. [doi: 10.2196/mhealth.8917]

38. Hetrick SE, Robinson J, Burge E, Blandon R, Mobilio B, Rice SM, et al. Youth Codesign of a Mobile Phone App to Facilitate Self-Monitoring and Management of Mood Symptoms in Young People With Major Depression, Suicidal Ideation, and Self-Harm. JMIR Ment Health 2018 Jan 23;5(1):e9 [FREE Full text] [doi: 10.2196/mental.9041] [Medline: 29362208]

39. Cochrane. Feasibility trial of ClinTouch-CareLoop Enhanced Management. Cochrane. 2014. URL: https://www. cochranelibrary.com/central/doi/10.1002/central/CN-01857649/full [accessed 2020-04-23]

40. Kodama T, Syouji H, Takaki S, Fujimoto H, Ishikawa S, Fukutake M, et al. Text Messaging for Psychiatric Outpatients: Effect on Help-Seeking and Self-Harming Behaviors. J Psychosoc Nurs Ment Health Serv 2016 Apr;54(4):31-37. [doi: 10.3928/02793695-20160121-01] [Medline: 27042926]

41. Mackie C, Dunn N, MacLean S, Testa V, Heisel M, Hatcher S. A qualitative study of a blended therapy using problem solving therapy with a customised smartphone app in men who present to hospital with intentional self-harm. Evid Based Ment Health 2017 Nov;20(4):118-122. [doi: 10.1136/eb-2017-102764] [Medline: 29030503]

42. Madsen LB, Eddleston M, Hansen KS, Pearson M, Agampodi S, Jayamanne S, et al. Cost-effectiveness analyses of self-harm strategies aimed at reducing the mortality of pesticide self-poisonings in Sri Lanka: a study protocol. BMJ Open 2015 Feb 27;5(2):e007333 [FREE Full text] [doi: 10.1136/bmjopen-2014-007333] [Medline: 25724984]

43. Marasinghe RB, Edirippulige S, Kavanagh D, Smith A, Jiffry MTM. Effect of mobile phone-based psychotherapy in suicide prevention: a randomized controlled trial in Sri Lanka. J Telemed Telecare 2012 Apr;18(3):151-155. [doi:

10.1258/jtt.2012.SFT107] [Medline: 22362830] 
44. Cochrane. Text Message Intervention to Reduce Repeat Self-harm. Cochrane. 2013. URL: https://www.cochranelibrary.com/ central/doi/10.1002/central/CN-01541374/full [accessed 2020-04-23]

45. Cochrane. Effects of Community-based Caring Contact on Post-discharge Young Adults With Self-harm. Cochrane. 2017. URL: https://www.cochranelibrary.com/central/doi/10.1002/central/CN-01562629/full [accessed 2020-04-23]

46. Cochrane. The BEACON Study: smartphone-Assisted Problem-Solving Therapy in Men Presenting to the ED With Self-Harm (Protocol A). Cochrane. 2018. URL: https://www.cochranelibrary.com/central/doi/10.1002/central/CN-01567843/ full [accessed 2020-04-23]

47. Owens C, Charles N. Implementation of a text-messaging intervention for adolescents who self-harm (TeenTEXT): a feasibility study using normalisation process theory. Child Adolesc Psychiatry Ment Health 2016;10:14 [FREE Full text] [doi: 10.1186/s13034-016-0101-z] [Medline: 27354855]

48. Rizvi SL, Hughes CD, Thomas MC. The DBT Coach mobile application as an adjunct to treatment for suicidal and self-injuring individuals with borderline personality disorder: A preliminary evaluation and challenges to client utilization. Psychol Serv 2016 Dec;13(4):380-388. [doi: 10.1037/ser0000100] [Medline: 27797571]

49. Sayal K, Roe J, Ball H, Atha C, Kaylor-Hughes C, Guo B, et al. Feasibility of a randomised controlled trial of remotely delivered problem-solving cognitive behaviour therapy versus usual care for young people with depression and repeat self-harm: lessons learnt (e-DASH). BMC Psychiatry 2019 Jan 24;19(1):42. [doi: 10.1186/s12888-018-2005-3] [Medline: $\underline{30678674}$ ]

50. Andreasson K, Krogh J, Bech P, Frandsen H, Buus N, Stanley B, et al. MYPLAN -mobile phone application to manage crisis of persons at risk of suicide: study protocol for a randomized controlled trial. Trials 2017 Dec 11;18(1):171 [FREE Full text] [doi: 10.1186/s13063-017-1876-9] [Medline: 28399909]

51. Skovgaard LJL, Frandsen H, Erlangsen A. MYPLAN - A Mobile Phone Application for Supporting People at Risk of Suicide. Crisis 2016 May;37(3):236-240. [doi: 10.1027/0227-5910/a000371] [Medline: 26831213]

52. Stevens GJ, Hammond TE, Brownhill S, Anand M, de la Riva A, Hawkins J, et al. SMS SOS: a randomized controlled trial to reduce self-harm and suicide attempts using SMS text messaging. BMC Psychiatry 2019 Apr 18;19(1):117 [FREE Full text] [doi: 10.1186/s12888-019-2104-9] [Medline: 30999952]

53. Szlyk HS, Roth KB, García-Perdomo V. Engagement With Crisis Text Line Among Subgroups of Users Who Reported Suicidality. Psychiatr Serv 2020 Apr 01;71(4):319-327. [doi: 10.1176/appi.ps.201900149] [Medline: 31795857]

54. Werner-Seidler A, Huckvale K, Larsen ME, Calear AL, Maston K, Johnston L, et al. A trial protocol for the effectiveness of digital interventions for preventing depression in adolescents: The Future Proofing Study. Trials 2020 Jan 02;21(1):2 [FREE Full text] [doi: 10.1186/s13063-019-3901-7] [Medline: 31898512]

55. Bjureberg J, Sahlin H, Hedman-Lagerlöf E, Gratz KL, Tull MT, Jokinen J, et al. Extending research on Emotion Regulation Individual Therapy for Adolescents (ERITA) with nonsuicidal self-injury disorder: open pilot trial and mediation analysis of a novel online version. BMC Psychiatry 2018 Oct 11;18(1):326 [FREE Full text] [doi: 10.1186/s12888-018-1885-6] [Medline: 30305103]

56. Van Voorhees BW, Fogel J, Reinecke MA, Gladstone T, Stuart S, Gollan J, et al. Randomized clinical trial of an Internet-based depression prevention program for adolescents (Project CATCH-IT) in primary care: 12-week outcomes. J Dev Behav Pediatr 2009 Feb;30(1):23-37 [FREE Full text] [doi: 10.1097/DBP.0b013e3181966c2a] [Medline: 19194326]

57. Evans MO, Morgan HG, Hayward A, Gunnell DJ. Crisis telephone consultation for deliberate self-harm patients: effects on repetition. Br J Psychiatry 1999 Jul;175:23-27. [doi: 10.1192/bjp.175.1.23] [Medline: 10621764]

58. Eylem O, van SA, Bhui K, Kerkhof AJFM. Protocol: Reducing suicidal ideation among Turkish migrants in the Netherlands and in the UK: effectiveness of an online intervention. Int Rev Psychiatry 2015 Feb;27(1):72-81. [doi: 10.3109/09540261.2014.996121] [Medline: 25747026]

59. van Spijker BAJ, van Straten A, Kerkhof AJFM. The effectiveness of a web-based self-help intervention to reduce suicidal thoughts: a randomized controlled trial. Trials 2010;11:25 [FREE Full text] [doi: 10.1186/1745-6215-11-25] [Medline: 20214777]

60. Hooley JM, Fox KR, Wang SB, Kwashie AND. Novel online daily diary interventions for nonsuicidal self-injury: a randomized controlled trial. BMC Psychiatry 2018 Aug 22;18(1):264 [FREE Full text] [doi: 10.1186/s12888-018-1840-6] [Medline: 30134866]

61. Lederer N, Grechenig T, Baranyi R. unCUT: bridging the gap from paper diary cards towards mobile electronic monitoring solutions in borderline and self-injury. : SeGAH; 2014 Presented at: 2014 IEEE 3nd International Conference on Serious Games and Applications for Health (SeGAH); 14-16 May 2014; Brazil. [doi: 10.1109/segah.2014.7067092]

62. Mühlmann C, Madsen T, Hjorthøj C, Kerkhof A, Nordentoft M, Erlangsen A. The Self-help Online against Suicidal thoughts (SOS) trial: study protocol for a randomized controlled trial. Trials 2017 Jan 28;18(1):45 [FREE Full text] [doi: 10.1186/s13063-017-1794-x] [Medline: 28129783]

63. van Spijker BAJ, van Straten A, Kerkhof AJFM. Effectiveness of online self-help for suicidal thoughts: results of a randomised controlled trial. PLoS One 2014;9(2):e90118 [FREE Full text] [doi: 10.1371/journal.pone.0090118] [Medline: $\underline{24587233}$ ] 
64. McManama OKH, LeCloux M, Ross A, Gironda C, Wharff EA. A Pilot Study of the Acceptability and Usability of a Smartphone Application Intervention for Suicidal Adolescents and Their Parents. Arch Suicide Res 2016 May 2:1-11. [doi: 10.1080/13811118.2016.1182094] [Medline: 27136115]

65. Helweg-Jørgensen S, Beck Lichtenstein M, Fruzzetti AE, Møller Dahl C, Pedersen SS. Daily Self-Monitoring of Symptoms and Skills Learning in Patients With Borderline Personality Disorder Through a Mobile Phone App: Protocol for a Pragmatic Randomized Controlled Trial. JMIR Res Protoc 2020 May 25;9(5):e17737 [FREE Full text] [doi: 10.2196/17737] [Medline: 32449690]

66. Duan S, Wang H, Wilson A, Qiu J, Chen G, He Y, et al. Developing a Text Messaging Intervention to Reduce Deliberate Self-Harm in Chinese Adolescents: Qualitative Study. JMIR Mhealth Uhealth 2020 Jun 11;8(6):e16963 [FREE Full text] [doi: 10.2196/16963] [Medline: 32392173]

67. Kerner B, Carlson M, Eskin CK, Tseng C, Ho JG, Zima B, et al. Trends in the utilization of a peer-supported youth hotline. Child Adolesc Ment Health 2020 May 25:65-72. [doi: 10.1111/camh.12394] [Medline: $\underline{32449589]}$

68. Al-Sharifi A, Krynicki CR, Upthegrove R. Self-harm and ethnicity: A systematic review. Int J Soc Psychiatry 2015 Sep;61(6):600-612. [doi: 10.1177/0020764015573085] [Medline: 25783961]

69. Rimes K, Goodship N, Ussher G, Baker D, West E. Non-binary and binary transgender youth: Comparison of mental health, self-harm, suicidality, substance use and victimization experiences. Int J Transgenderism. (2-3) 2019;20:230-240. [doi: 10.4324/9781003015888-11] [Medline: $\underline{\text { 32999609] }}$

70. Glenn CR, Klonsky ED. One-year test-retest reliability of the Inventory of Statements about Self-Injury (ISAS). Assessment 2011 Sep;18(3):375-378. [doi: 10.1177/1073191111411669] [Medline: 21665881]

71. Ofcom. Communications Market Report. Ofcom. 2018. URL: https://www.ofcom.org.uk/ data/assets/pdf file/0022/ 117256/CMR-2018-narrative-report.pdf [accessed 2020-08-11]

72. Hunkin H, King DL, Zajac IT. Perceived acceptability of wearable devices for the treatment of mental health problems. J Clin Psychol 2020 Jun;76(6):987-1003. [doi: 10.1002/jclp.22934] [Medline: $\underline{\text { 32022908] }}$

73. Gartner. Gartner Says Worldwide Wearable Device Sales to Grow 26 Percent in 2019. Gartner. 2018 Nov 29. URL: https:/ /www.gartner.com/en/newsroom/press-releases/ 2018-11-29-gartner-says-worldwide-wearable-device-sales-to-grow-\#: :text=Gartner\%2C\%20Inc. ,billion\%20will\%20be\%20on\%20smartwatches [accessed 2020-08-11]

74. Nicholas J, Larsen ME, Proudfoot J, Christensen H. Mobile Apps for Bipolar Disorder: A Systematic Review of Features and Content Quality. J Med Internet Res 2015;17(8):e198 [FREE Full text] [doi: 10.2196/jmir.4581] [Medline: 26283290]

75. Willig C. Introducing qualitative research in psychology: Adventures in theory and method. Buckingham \& Philadelphia: Open University Press; 2008.

76. Mason J. Qualitative Researching. In: Qualitative Researching. London: SAGE Publications; 2012.

77. Owens C, Fox F, Redwood S, Davies R, Foote L, Salisbury N, et al. Measuring outcomes in trials of interventions for people who self-harm: qualitative study of service users' views. BJPsych Open 2020 Feb 12;6(2):e22 [FREE Full text] [doi: 10.1192/bjo.2019.93] [Medline: 32046813]

78. Cliffe B, Croker A, Denne M, Stallard P. Clinicians' use of and attitudes towards technology to provide and support interventions in child and adolescent mental health services. Child Adolesc Ment Health 2020 May;25(2):95-101. [doi: 10.1111/camh.12362] [Medline: $\underline{32307835]}$

79. Hollis C, Falconer CJ, Martin JL, Whittington C, Stockton S, Glazebrook C, et al. Annual Research Review: Digital health interventions for children and young people with mental health problems: a systematic and meta-review. J Child Psychol Psychiatry 2016 Dec 10:474-503. [doi: 10.1111/jepp.12663] [Medline: 27943285]

80. Aguilera A. Digital Technology and Mental Health Interventions: Opportunities and Challenges. Arbor 2015 May 12;191(771):a210. [doi: 10.3989/arbor.2015.771n1012]

81. Greaves F, Joshi I, Campbell M, Roberts S, Patel N, Powell J. What is an appropriate level of evidence for a digital health intervention? Lancet 2019 Dec 22;392(10165):2665-2667. [doi: 10.1016/S0140-6736(18)33129-5] [Medline: 30545779]

82. Pagoto S, Bennett GG. How behavioral science can advance digital health. Transl Behav Med 2013 Sep;3(3):271-276 [FREE Full text] [doi: 10.1007/s13142-013-0234-z] [Medline: 24073178]

83. Morrison K. Randomised Controlled Trials for Evidence-based Education: Some Problems in Judging 'What Works'. Evaluation \& Research in Education 2001 Oct;15(2):69-83. [doi: 10.1080/09500790108666984]

84. Raghavan R, Munson MR, Le C. Toward an Experimental Therapeutics Approach in Human Services Research. Psychiatr Serv 2019 Dec 01;70(12):1130-1137 [FREE Full text] [doi: 10.1176/appi.ps.201800577] [Medline: $\underline{31500543}$ ]

\section{Abbreviations}

mHealth: mobile health

RCT: randomized controlled trial 
Edited by R Kukafka; submitted 19.10.20; peer-reviewed by E Bailey, E Kleiman, B Zima; comments to author 05.12.20; revised version received 19.01.21; accepted 16.03.21; published 30.04.21

Please cite as:

Cliffe B, Tingley J, Greenhalgh I, Stallard P

mHealth Interventions for Self-Harm: Scoping Review

$J$ Med Internet Res 2021;23(4):e25140

URL: https://www.jmir.org/2021/4/e25140

doi: $10.2196 / 25140$

PMID:

CBethany Cliffe, Jessica Tingley, Isobel Greenhalgh, Paul Stallard. Originally published in the Journal of Medical Internet Research (https://www.jmir.org), 30.04.2021. This is an open-access article distributed under the terms of the Creative Commons Attribution License (https://creativecommons.org/licenses/by/4.0/), which permits unrestricted use, distribution, and reproduction in any medium, provided the original work, first published in the Journal of Medical Internet Research, is properly cited. The complete bibliographic information, a link to the original publication on https://www.jmir.org/, as well as this copyright and license information must be included. 